\title{
HOMOMORPHIC PREIMAGES OF GEOMETRIC PATHS
}

\author{
SAlly COCKBURN \\ Mathematics Department, Hamilton College \\ Clinton NY, USA \\ e-mail: scockbur@hamilton.edu
}

\begin{abstract}
A graph $G$ is a homomorphic preimage of another graph $H$, or equivalently $G$ is $H$-colorable, if there exists a graph homomorphism $f: G \rightarrow H$. A geometric graph $\bar{G}$ is a simple graph $G$ together with a straight line drawing of $G$ in the plane with the vertices in general position. A geometric homomorphism (respectively, isomorphism) $\bar{G} \rightarrow \bar{H}$ is a graph homomorphism (respectively, isomorphism) that preserves edge crossings (respectively, and non-crossings). The homomorphism poset $\mathcal{G}$ of a graph $G$ is the set of isomorphism classes of geometric realizations of $G$ partially ordered by the existence of injective geometric homomorphisms. A geometric graph $\bar{G}$ is $\mathcal{H}$-colorable if $\bar{G} \rightarrow \bar{H}$ for some $\bar{H} \in \mathcal{H}$. In this paper, we provide necessary and sufficient conditions for $\bar{G}$ to be $\mathcal{P}_{n}$-colorable for $n \geq 2$. Along the way, we also provide necessary and sufficient conditions for $\bar{G}$ to be $\mathcal{K}_{2,3}$-colorable.
\end{abstract}

Keywords: geometric graphs, graph homomorphisms.

2010 Mathematics Subject Classification: 05C60, 05C62, 05C38.

\section{REFERENCES}

[1] D.L. Boutin and S. Cockburn, Geometric graph homomorphisms, J. Graph Theory 69 (2012) 97-113.

doi:10.1002/jgt.20566

[2] D.L. Boutin, S. Cockburn, A.M. Dean and A.M. Margea, Posets of geometric graphs, Ars Math. Contemp. 5 (2012) 269-288.

[3] S. Cockburn, The homomorphism poset of $K_{3,3}$, (2013). arxiv: 1306.5732

[4] S. Cockburn, Homomorphism preimages of geometric cycles, J. Combin. Math. Combin. Comput., to appear. 
[5] S. Cockburn and Y. Song, The homomorphism poset of $K_{2, n}$, Australas. J. Combin. 57 (2013) 79-108.

[6] H.Harborth, Parity of numbers of crossings for complete n-partite graphs, Math. Slovaca 26 (1976) 77-95.

[7] P. Hell and J. Nešetřil, Graphs and Homomorphisms (Oxford University Press, Oxford, 2004).

doi:10.1093/acprof:oso/9780198528173.001.0001

[8] P. Hell and J. Nešetřil, On the complexity of H-coloring, J. Combin. Theory Ser. B 48 (1990) 92-110

doi:10.1016/0095-8956(90)90132-J

[9] P. Hell, H. Zhou and X. Zhu, Homomorphisms to oriented cycles, Combinatorica 13 (1993) 421-433. doi:10.1007/BF01303514

[10] P. Hell, H. Zhou and X. Zhu, On homomorphisms to acyclic local tournaments, J. Graph Theory 20 (1995) 467-471.

doi:10.1002/jgt.3190200410

[11] P. Hell and X. Zhu, Homomorphisms to oriented paths, Discrete Math. 132 (1994) $107-114$ doi:10.1016/0012-365X(94)90235-6

[12] P. Hell and X. Zhu, The existence of homomorphisms to oriented cycles, SIAM J. Discrete Math. 8 (1995) 208-222. doi:10.1137/S0895480192239992

[13] H.A. Maurer, A. Salomaa and D. Wood, Colorings and interpretations: a connection between graphs and grammar forms, Discrete Appl. Math. 3 (1981) 119-135. doi:10.1016/0166-218X(81)90037-8

[14] H. Zhou, Characterization of the homomorphic preimages of certain oriented cycles, SIAM J. Discrete Math. 6 (1993) 87-99.

doi:10.1137/0406007

Received 25 September 2015

Revised 15 October 2016

Accepted 11 January 2017 